\title{
Effect of Nonmagnetic Impurities on the Magnetic Resonance Peak in $\mathrm{YBa}_{2} \mathrm{Cu}_{3} \mathrm{O}_{7}$
}

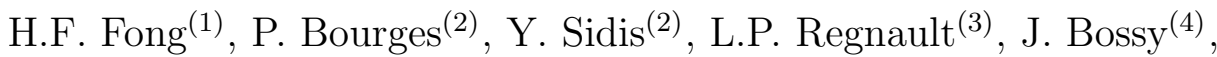
A. Ivanov ${ }^{(5)}$, D.L. Milius ${ }^{(6)}$, I.A. Aksay ${ }^{(6)}$, and B. Keimer ${ }^{(1,7)}$

1 Department of Physics, Princeton University, Princeton, NJ 08544 USA

2 Laboratoire Léon Brillouin, CEA-CNRS, CE Saclay, 91191 Gif sur Yvette, France

3 CEA Grenoble, Département de Recherche Fondamentale sur la matière Condensée, 38054 Grenoble cedex 9, France

4 CNRS-CRTBT, BP 156, 38042 Grenoble Cedex 9, France

5 Institut Laue-Langevin, 156X, 38042 Grenoble Cedex 9, France

6 Department of Chemical Engineering, Princeton University, Princeton, NJ 08544 USA

7 Max-Planck-Institut für Festkörperforschung, D-70569 Stuttgart, Germany

\begin{abstract}
The magnetic excitation spectrum of a $\mathrm{YBa}_{2} \mathrm{Cu}_{3} \mathrm{O}_{7}$ crystal containing $0.5 \%$ of nonmagnetic (Zn) impurities has been determined by inelastic neutron scattering. Whereas in the pure system a sharp resonance peak at $E \simeq 40 \mathrm{meV}$ is observed exclusively below the superconducting transition temperature $\mathrm{T}_{\mathrm{c}}$, the magnetic response in the Zn-substituted system is broadened significantly and vanishes at a temperature much higher than $\mathrm{T}_{\mathrm{c}}$. The energyintegrated spectral weight observed near $\mathbf{q}=(\pi, \pi)$ increases with Zn substitution, and only about half of the spectral weight is removed at $\mathrm{T}_{\mathrm{c}}$.
\end{abstract}


The magnetic resonance peak is a sharp collective excitation at an energy of $40 \mathrm{meV}$ and wavevector $\mathbf{q}_{0}=(\pi, \pi)$ in the superconducting state of $\mathrm{YBa}_{2} \mathrm{Cu}_{3} \mathrm{O}_{7}$ [1]-[7]. The peak is also observed at lower energies in underdoped $\mathrm{YBa}_{2} \mathrm{Cu}_{3} \mathrm{O}_{6+\mathrm{x}}$ [8, 9, 10]. As the existence of this peak requires d-wave superconductivity, it demonstrates that magnetic neutron scattering is a phase sensitive probe of superconductivity [4]. The peak also provides important clues to the microscopic mechanism of high temperature superconductivity: It does not appear in the Lindhard susceptibility of a noninteracting band metal [11], and the interactions responsible for the enhancement of the band susceptibility are presumably the same as the ones that drive superconductivity. Several enhancement mechanisms have thus been suggested: band structure singularities [12], antiferromagnetic interactions [13], and interlayer tunneling [14]. Other models of the resonance peak [15, 16, 17] appeal directly to the parent antiferromagnetic insulator $\mathrm{YBa}_{2} \mathrm{Cu}_{3} \mathrm{O}_{6}$, where low energy spin waves of spectral weight comparable to the resonance peak are observed near $\mathbf{q}_{0}=(\pi, \pi)$.

Clearly, more experimental data are needed to differentiate between these theories. Here we present neutron scattering measurements of the spin dynamics of a $\mathrm{YBa}_{2} \mathrm{Cu}_{3} \mathrm{O}_{7}$ single crystals in which a small number of nonmagnetic zinc ions replace copper ions. Zn substitution introduces minimal structural disorder, substitutes for copper in the $\mathrm{CuO}_{2}$ planes [18], and does not modify the hole concentration substantially [19]. The Zn ions are known to induce local magnetic moments on neighboring $\mathrm{Cu}$ sites [20] which are associated with low energy magnetic excitations [21, 22, 23]. It has further been shown that Zn impurities scatter conduction electrons near the unitary limit and rapidly suppress the superconducting transition temperature, $\mathrm{T}_{\mathrm{c}}$ [24]. The new data reported here demonstrate a broadening of the spin excitation spectrum in the presence of a minute amount of $\mathrm{Zn}$ impurities. Instead of disappearing in the normal state as in zinc-free $\mathrm{YBa}_{2} \mathrm{Cu}_{3} \mathrm{O}_{7}$, the broadened intensity now persists well above $\mathrm{T}_{\mathrm{c}}$. These results are surprising and were not anticipated by any of the theoretical models of the resonance peak.

A single crystal of composition $\mathrm{YBa}_{2}\left(\mathrm{Cu}_{0.995} \mathrm{Zn}_{0.005}\right)_{3} \mathrm{O}_{7}$ and volume $1.7 \mathrm{~cm}^{3}$ was prepared by a method described previously [6]. The crystal was annealed under oxygen flow at $600^{\circ} \mathrm{C}$ for 14 days, a procedure that resulted in a $T_{c}=93 \mathrm{~K}$ in Zn-free crystals synthesized by the same method [6]. After the heat treatment, the crystal showed $\mathrm{T}_{\mathrm{c}}=87 \mathrm{~K}$ width a width of 
about $5 \mathrm{~K}$, consistent with earlier reports on Zn-substituted, fully oxygenated $\mathrm{YBa}_{2} \mathrm{Cu}_{3} \mathrm{O}_{7}$ 24.

The measurements were taken at the IN8 triple axis spectrometer at the Institut Laue Langevin, Grenoble, France. Preliminary data were also taken at the BT2 spectrometer at the NIST research reactor. The IN8 beam optics included a vertically focusing $\mathrm{Cu}$ (111) monochromator, and a horizontally focusing pyrolytic graphite (002) analyser which selected a fixed final energy of $35 \mathrm{meV}$. A pyrolytic graphite filter was inserted into the scattered beam in order to eliminate higher-order contamination. The sample was attached to the cold finger of a closed cycle helium refrigerator mounted on a two-circle goniometer. Data were taken with the crystal in two different orientations where wave vectors of the forms $\mathbf{Q}=(H, H, L)$ and $(3 H, H, L)$ were accessible. [Throughout this article, the wave vector $\mathbf{Q}=(H, K, L)$ is indexed in units of the reciprocal lattice vectors $2 \pi / a \sim 2 \pi / b \sim 1.63 \AA^{-1}$ and $2 \pi / c \sim 0.53 \AA^{-1}$. In this notation, the $(\pi, \pi)$ point corresponds to $\left(\frac{h}{2}, \frac{k}{2}\right)$ with $h$ and $k$ integers.]

As described in detail elsewhere [5, [6], the imaginary part of the dynamical magnetic susceptibility, $\chi^{\prime \prime}(\mathbf{Q}, \omega)$, can be separated from phonon scattering with the aid of lattice vibrational calculations, and by studying the momentum, temperature and doping dependence of the neutron scattering cross section. In pure (Zn-free) $\mathrm{YBa}_{2} \mathrm{Cu}_{3} \mathrm{O}_{6+\mathrm{x}}$, this procedure was verified by measurements with polarized neutron beams for some scattering configurations in the energy range covered by the present study, $10 \mathrm{meV}$ through $50 \mathrm{meV}$ [6, 9]. Since the changes in the phonon spectrum induced by $0.5 \%$ Zn-substitution are insignificant, the data analysis procedures developed for pure $\mathrm{YBa}_{2} \mathrm{Cu}_{3} \mathrm{O}_{6+\mathrm{x}}$ carry over directly to the sample investigated here.

Fig. 1 shows representative constant-energy scans taken in the $(H, H, L)$ zone. As in pure $\mathrm{YBa}_{2} \mathrm{Cu}_{3} \mathrm{O}_{7}$, the magnetic scattering is confined to a window around $40 \mathrm{meV}$ and $\mathbf{q}_{0}=(\pi, \pi)$, with no magnetic scattering observed above background at low energies. The magnetic scattering around $40 \mathrm{meV}$ also exhibits the sinusoidal modulation in $L$ discussed in detail previously [回- [6], 25]. An upper limit of $1 / 3$ of the maximum intensity can be placed on the intensity at the minimum of the modulation, as in the pure system. These and similar scans taken in the $(3 H, H, L)$ zone were put on an absolute unit scale by normalizing 
to the phonon spectrum following Ref. [6], using the same definition of the spin susceptibility as Ref. 26] (Fig. 2a). The constant-energy scans were fitted to Gaussian profiles whose amplitude is plotted in Fig. $2 \mathrm{~b}$ as a function of energy.

The overall shape of the magnetic spectrum of $\mathrm{YBa}_{2}\left(\mathrm{Cu}_{0.995} \mathrm{Zn}_{0.005}\right)_{3} \mathrm{O}_{7}$, being concentrated around a characteristic energy $40 \mathrm{meV}$ and wave vector $\mathbf{q}_{0}=(\pi, \pi)$, is clearly reminiscent of the resonance peak in the pure system. The spectral weight increases at low temperatures, as does the resonance peak in pure $\mathrm{YBa}_{2} \mathrm{Cu}_{3} \mathrm{O}_{7}$. However, there are also substantial differences between the pure and Zn-substituted systems. First, while the magnetic resonance peak in the pure system is very sharp in energy, the magnetic response in the Zn-substituted sample is substantially broadened. This is apparent in the temperature difference spectrum (Fig. 2c), which gives a more detailed picture of the energy range near $40 \mathrm{meV}$. (In order to obtain better counting statistics, the high temperature cross section at constant wave vector was subtracted from the low temperature cross section at the same wave vector, without a full Q-scan at each energy. Since the phonon scattering is temperature independent in this energy and temperature range, only magnetic scattering contributes to the difference spectrum.) The full width at half maximum of the difference spectrum is $\Delta E \sim 10 \mathrm{meV}$, much broader than the instrumental resolution $(\sim 5 \mathrm{meV})$, yielding an intrinsic energy width of $\sim 8.5 \mathrm{meV}$. The width of the unsubtracted spectrum in Fig. $2 \mathrm{~b}$ is also consistent with this value. Within the errors, the full width at half maximum in Q-space, $\Delta Q \sim 0.25 \AA^{-1}$ at $E=39 \mathrm{meV}$, is identical to the resonance width in the pure system (Fig. 1).

A series of constant-energy scans at energies $39 \mathrm{meV}$ and $35 \mathrm{meV}$ were carried out at temperatures up to $\sim 300 \mathrm{~K}$ and fitted to Gaussian profiles. The fitted amplitudes for 39 meV are plotted in Fig. 3; the data for $35 \mathrm{meV}$ track those of Fig. 3 to within the errors. This figure fully reveals an even more dramatic difference of the spectra in the pure and Znsubstituted systems, already indicated in Figs. 2a and b. Whereas the magnetic response in the pure system (restricted to a single resonance peak) disappears in the normal state [4, 5, 6], Fig. 3 shows that in $\mathrm{YBa}_{2}\left(\mathrm{Cu}_{0.995} \mathrm{Zn}_{0.005}\right)_{3} \mathrm{O}_{7}$ the magnetic spectral weight actually persists up to $\sim 250 \mathrm{~K}$. Furthermore, in both underdoped and optimally doped systems, the magnetic resonance peak follows a sharp, order parameter-like curve below $\mathrm{T}_{\mathrm{c}}$ (Refs. [5]- [10]). 
By contrast, there is at most a weak inflection point near $\mathrm{T}_{\mathrm{c}}$ in the Zn-substituted system. The influence of superconductivity on the spin excitations, which is so clearly apparent in pure $\mathrm{YBa}_{2} \mathrm{Cu}_{3} \mathrm{O}_{6+\mathrm{x}}$, is thus almost completely obliterated by $0.5 \% \mathrm{Zn}$ substitution.

A further important comparison between the pure and Zn-substituted materials is made possible by the absolute unit calibration. Since the resonance peak in the pure system is very sharp and comparable to the instrumental energy resolution, the appropriate quantity to compare is the energy-integrated magnetic spectral weight, $\int d \omega \chi^{\prime \prime}\left(\mathbf{q}_{0}, \omega\right)$, in the energy range probed by the neutron experiment. This quantity is $2.2 \pm 0.5 \mu_{B}^{2}$ at low temperatures in $\mathrm{YBa}_{2}\left(\mathrm{Cu}_{0.995} \mathrm{Zn}_{0.005}\right)_{3} \mathrm{O}_{7}$, as compared to $1.6 \pm 0.5 \mu_{B}^{2}$ in $\mathrm{YBa}_{2} \mathrm{Cu}_{3} \mathrm{O}_{7}$ [27]. (Note, however, that in $\mathrm{YBa}_{2}\left(\mathrm{Cu}_{0.995} \mathrm{Zn}_{0.005}\right)_{3} \mathrm{O}_{7}$ only half of this intensity is removed upon heating to $\mathrm{T}_{\mathrm{c}}$, whereas in the pure system no magnetic intensity is observable above $T_{c}$.) As nonmagnetic impurities are added, the total energy-integrated spectral weight around $(\pi, \pi)$ therefore increases in the energy range probed by the neutron experiment, implying that zinc restores antiferromagnetic correlations. In this respect, the Zn-substituted system resembles the underdoped pure system $(\mathrm{x}<0.95)$ where a normal state antiferromagnetic contribution exists [1, 3, 7]. Surprisingly, in the Zn-doped system this additional intensity appears in the same energy and wave vector range as the resonance peak in the pure system.

It is also interesting to compare the present data to previous neutron scattering work on more heavily Zn substituted cuprate superconductors [21]-23]. The enhanced low energy excitations near $\mathbf{q}_{0}=(\pi, \pi)$ reported for these materials were not observed in our very lightly Zn-substituted sample (bottom panel in Fig. 1). However, at higher energies a $2 \%$ Zn-substituted, fully oxygenated $\mathrm{YBa}_{2} \mathrm{Cu}_{3} \mathrm{O}_{7}$ sample investigated by Sidis et al. [21] exhibits a spectral distribution closely similar to the one shown in Fig. 2. The temperature evolution of the magnetic intensity [7] is also consistent with the one reported here (Fig. 3).

In summary, the effect of substituting one out of 200 copper atoms by nonmagnetic impurities is dramatic. The total spectral weight near $\mathbf{q}_{0}=(\pi, \pi)$ actually increases and persists to higher temperatures while remaining centered around $40 \mathrm{meV}$. On the other hand, the characteristic features of the resonance peak (i.e., its sharpness in energy and its coupling to superconductivity) are obliterated. It is worth noting that in the underdoped regime, where the normal-state susceptibility is also enhanced with respect to $\mathrm{YBa}_{2} \mathrm{Cu}_{3} \mathrm{O}_{7}$, 
the resonance peak remains sharp and coupled to superconductivity [8]-10]. This aspect thus seems to be a manifestation of a delicate coherence that is very easily disrupted by disorder. While none of the theories of the resonance peak [11-16 has anticipated this behavior, it is reminiscent of the extreme susceptibility of collective-singlet ground states in quasi-one dimensional systems (realized, for instance, in spin-Peierls and spin ladder materials) to nonmagnetic impurities. A microscopic analogy between both systems was pointed out by Fukuyama and coworkers [28], but its consequences for the spin excitations have not yet been evaluated. Viewed from a different angle, a gradual buildup of spectral weight below $\mathrm{T} \sim 250 \mathrm{~K}$ (as shown in Fig. 3) is also observed in underdoped $\mathrm{YBa}_{2} \mathrm{Cu}_{3} \mathrm{O}_{6+\mathrm{x}}$, where it is centered around a somewhat lower energy (20-30 meV) and goes along with the opening of the "spin pseudo-gap" [3, 26]. The strong temperature evolution in the normal states of both underdoped and disordered $\mathrm{YBa}_{2} \mathrm{Cu}_{3} \mathrm{O}_{6+\mathrm{x}}$ is obviously closely related to the resonance

peak and should be part of a comprehensive theoretical description of the spin dynamics of the cuprates.

\section{Acknowledgments}

We are grateful for technical assistance provided by D. Puschner. The work at Princeton University was supported by the National Science Foundation under Grant No. DMR9400362, and by the Packard and Sloan Foundations. 


\section{References}

[1] J. Rossat-Mignod et al., Physica C 185-189, 86 (1991).

[2] H.A. Mook et al., Phys. Rev. Lett. 70, 3490 (1993).

[3] L.P. Regnault et al., Physica C, 235-240, 59, (1994); Physica B, 213\&214, 48, (1995).

[4] H.F. Fong et al., Phys. Rev. Lett. 75, 316 (1995).

[5] P. Bourges, L.P. Regnault, Y. Sidis and C. Vettier, Phys. Rev. B 53, 876 (1996).

[6] H.F. Fong et al., Phys. Rev. B. 54, 6708 (1996).

[7] L.P. Regnault et al. in Neutron Scattering in Layered Copper-Oxide Superconductors, Edited by A. Furrer, (Kluwer, Amsterdam, 1998), p. 85.

[8] P. Dai et al., Phys. Rev. Lett 77, 5425 (1996).

[9] H.F. Fong, B. Keimer, D.L. Milius and I.A. Aksay, Phys. Rev. Lett. 78, 713 (1997).

[10] P. Bourges et al., Europhys. Lett. 38, 313 (1997).

[11] I.I. Mazin and V.M. Yakovenko, Phys. Rev. Lett. 75, 4134 (1995).

[12] N. Bulut and D.J. Scalapino, Phys. Rev. B 53, 5149 (1996); G. Blumberg, B.P. Stojkovic and M.V. Klein, ibid. 52, 15741 (1995); A.A. Abrikosov, ibid. 57, 8656 (1998).

[13] D.Z. Liu, Y. Zha and K. Levin, Phys. Rev. Lett. 75, 4130 (1995); F. Onufrieva, Physica C 251, 348 (1995); A.J. Millis and H. Monien, Phys. Rev. B 54, 16172 (1996).

[14] L. Yin, S. Chakravarty and P.W. Anderson, Phys. Rev. Lett. 78, 3559 (1997).

[15] E. Demler and S.C. Zhang, Phys. Rev. Lett. 75, 4126 (1995); S.C. Zhang, Science 275, 1089 (1997).

[16] Y. Zha, V. Barzykin and D. Pines, Phys. Rev. B 54, 7561 (1996); D.K. Morr and D. Pines, Report No. cond-mat/9805107. 
[17] F.F. Assaad and M. Imada, Report No. cond- mat/9711172.

[18] G. Xiao et al., Nature 332, 238 (1988); H. Maeda et al., Physica C 157, 483 (1989).

[19] H. Alloul et al., Phys. Rev. Lett. 67, 3140 (1991).

[20] V. A. Mahajan et al., Phys. Rev. Lett. 72, 3100 (1994).

[21] Y. Sidis et al., Phys. Rev. B 53, 6811 (1996).

[22] K. Kakurai et al., Phys. Rev. B 48, 3485 (1993).

[23] M. Matsuda et al., J. Phys. Soc. Jpn. 62, 443 (1993).

[24] T.R. Chien, Z.Z. Wang and N.P. Ong, Phys. Rev. Lett. 67, 2088 (1991); D.A. Bonn et al., Phys. Rev. B 50, 4051 (1994); Fukuzumi et al., Phys. Rev. Lett.76, 684 (1996).

[25] D. Reznik et al., Phys. Rev. B 53, R14741 (1996); S.M. Hayden et al., ibid. 54, R6905 (1996).

[26] P. Bourges et al., Phys. Rev. B 56, R11439 (1997).

[27] Note that if the susceptibility is energy integrated in the energy range probed by the experiment and averaged over the two-dimensional Brillouin zone, the resulting numbers, $\int d \omega \int d^{2} q \chi^{\prime \prime}\left(\mathbf{q}_{0}, \omega\right) / \int d^{2} q=0.058 \mu_{B}^{2}$ in $\mathrm{YBa}_{2}\left(\mathrm{Cu}_{0.995} \mathrm{Zn}_{0.005}\right)_{3} \mathrm{O}_{7}$ and $0.043 \mu_{B}^{2}$ in $\mathrm{YBa}_{2} \mathrm{Cu}_{3} \mathrm{O}_{7}$ come out much smaller than the corresponding number $\frac{\pi}{3} s(s+1) g^{2} \mu_{B}^{2}$ required by the total moment sum rule for an insulating $s=1 / 2$ antiferromagnet. In deriving these numbers, we used the full width at half maximum in momentum space, which is $0.25 \AA^{-1}$ in both materials. Note also that in underdoped materials where the spin excitation spectrum is much broader in energy, it is often convenient to quote the Brillouin zone averaged (local) susceptibility $\int d^{2} q \chi^{\prime \prime}\left(\mathbf{q}_{0}, \omega\right) / \int d^{2} q$ without integrating over energy [26].

[28] N. Nagaosa et al., J. Phys. Soc. Jpn. 65, 3724 (1996); H. Fukuyama, T. Tanimoto, and M. Saito, ibid. 65, 1182 (1996). 


\section{Figure Captions}

1. Constant-energy scans at $39 \mathrm{meV}$ and $10 \mathrm{meV}$ through $\mathbf{Q}=\left(\frac{1}{2}, \frac{1}{2}, L\right)$ for $\mathrm{YBa}_{2}\left(\mathrm{Cu}_{0.995} \mathrm{Zn}_{0.005}\right)_{3} \mathrm{O}_{7}$. The line in the upper panel is the results of a Gaussian fit. The bar gives the instrumental Q-resolution. Because of the good energy resolution $(\sim 5 \mathrm{meV})$, the $42.5 \mathrm{meV}$ phonon [4] makes only a weak contribution $(\leq 10 \%)$ to the upper scan.

2. a) Constant-energy scans through $(1.5,0.5,-1.7)$, background corrected and converted to absolute units. The bar gives the instrumental Q-resolution. b) Peak dynamical susceptibility at $\mathbf{q}_{0}=(\pi, \pi)$ extracted from fits to constant-energy profiles (panel a and Fig. 1). The line is a Gaussian with the same width as the difference spectrum in panel c. c) More detailed spectrum around $40 \mathrm{meV}$. The data around $100 \mathrm{~K}\left(>\mathrm{T}_{\mathrm{c}}\right)$ were subtracted from the low temperature data. The bar gives the instrumental energy resolution, and the line is the result of a fit to a Gaussian. All data are given in absolute units. $\mathrm{A} \sim 30 \%$ overall systematic error in the absolute unit calibration is not included in the error bars.

3. Temperature dependence of the dynamical susceptibility at $\mathbf{q}_{0}=(\pi, \pi)$ and at the peak energy of the spectrum $(\sim 39 \mathrm{meV})$, in absolute units. The closed circles are the fitted amplitudes of constant-energy scans, the open circles are the peak count rates. 


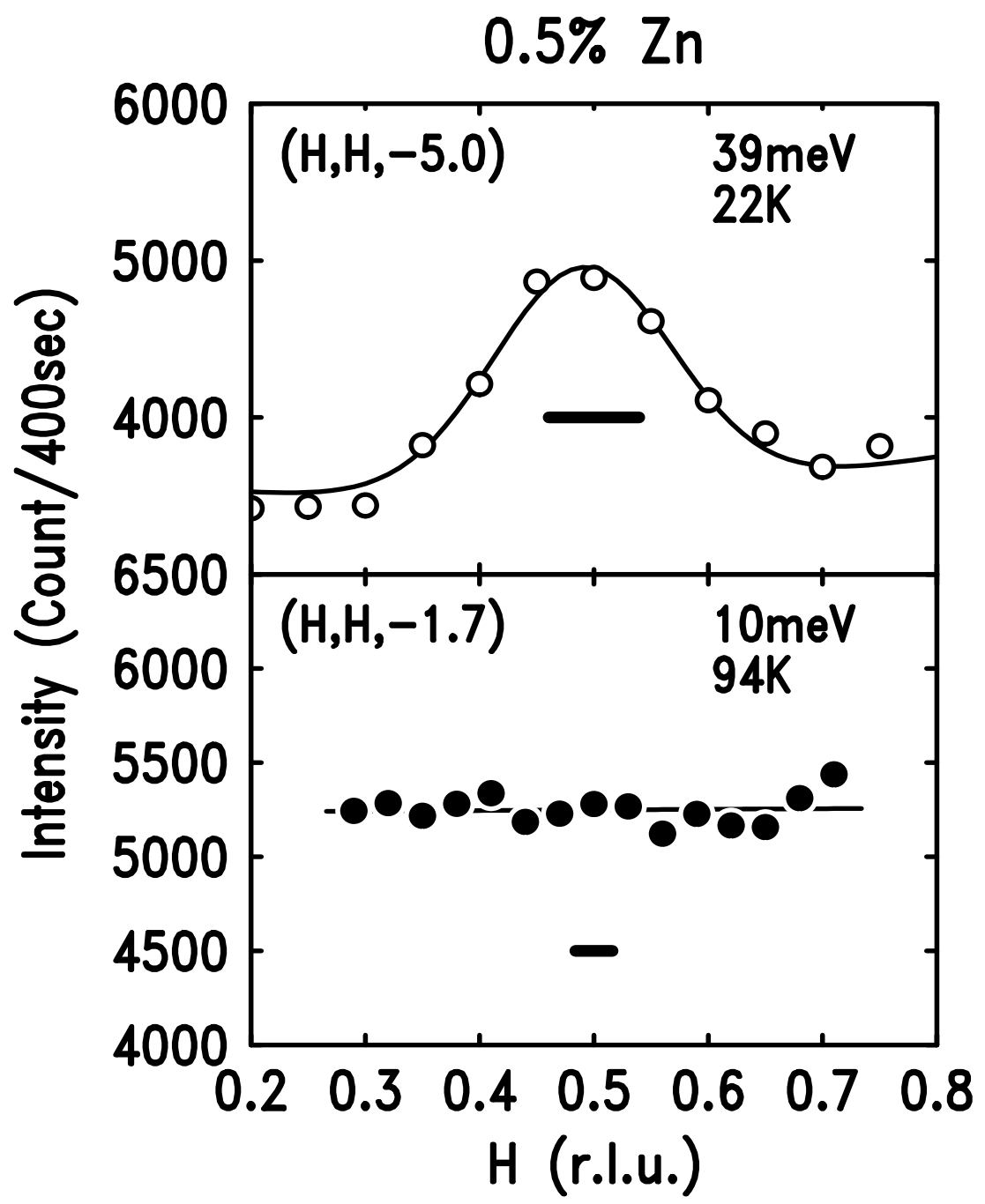



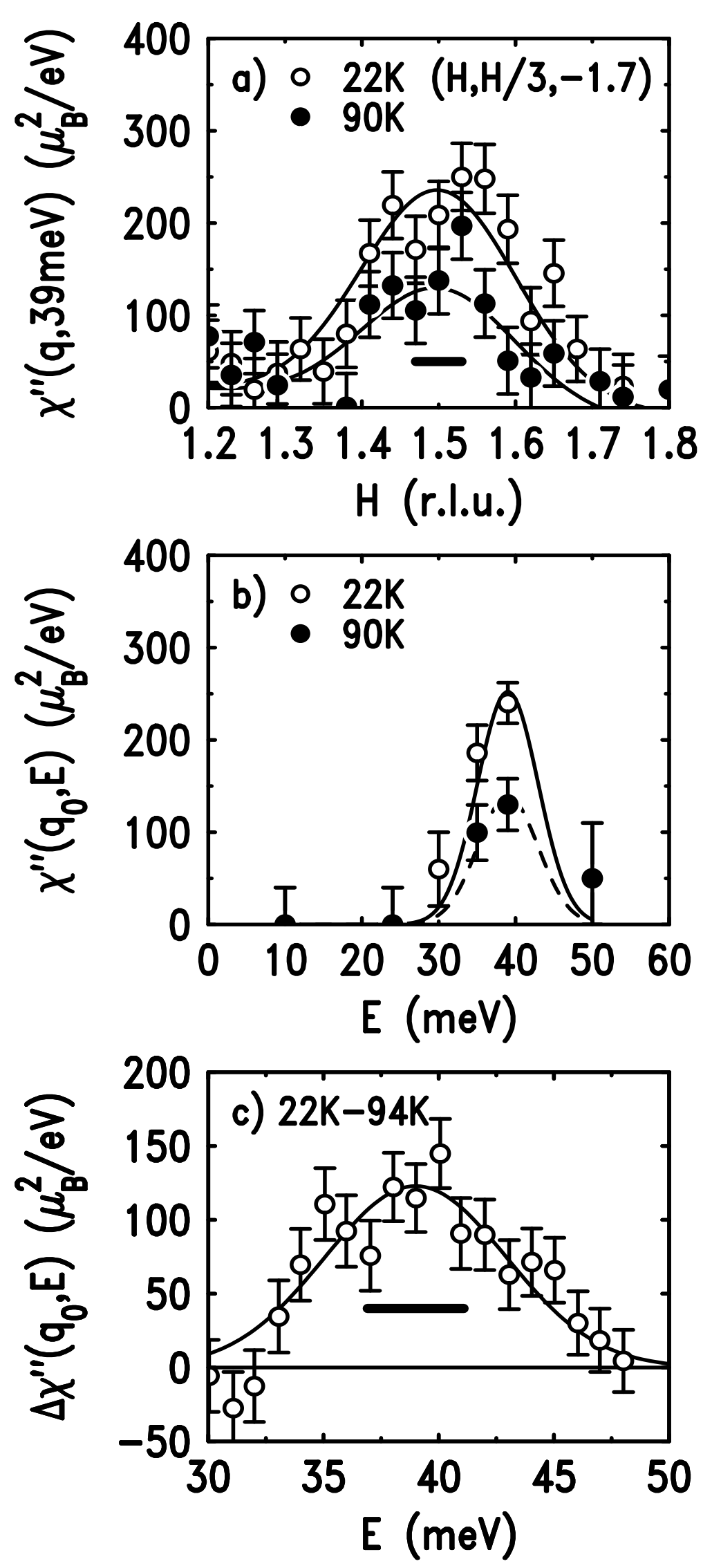


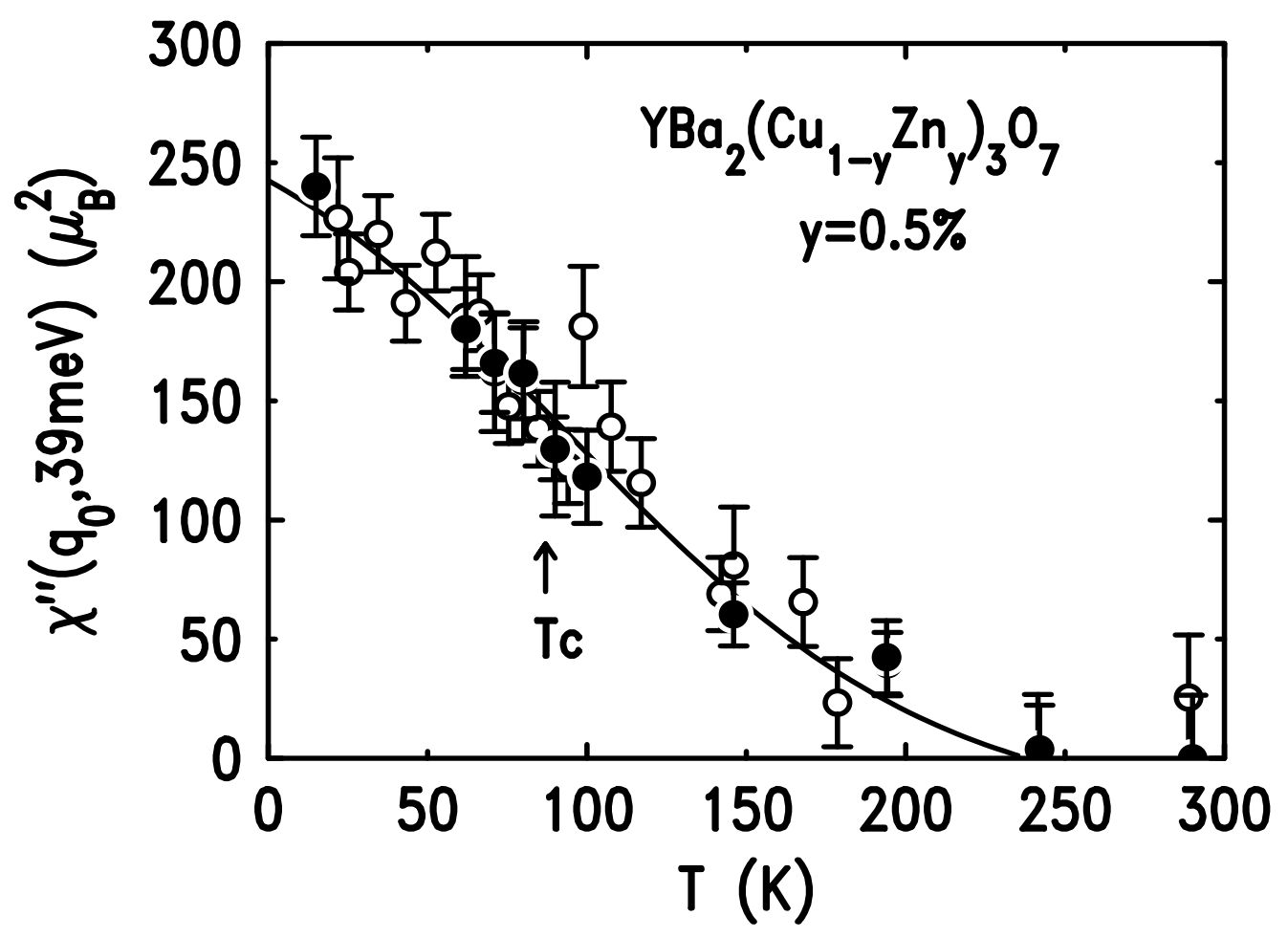

